\title{
One-neutron halo structure by the ratio method
}

\author{
P. Capel ${ }^{a, b, *}$, R. C. Johnson ${ }^{c, a}$, F. M. Nunes ${ }^{a}$ \\ ${ }^{a}$ National Superconducting Cyclotron Laboratory and Department of Physics and \\ Astronomy, Michigan State University, East Lansing, Michigan 48824, USA \\ ${ }^{b}$ Helmholtz-Insitut Mainz, Johannes Gutenberg-Universität, D-55128 Mainz, Germany \\ ${ }^{c}$ Department of Physics, University of Surrey, Guildford GU2 7XH, United Kingdom
}

\begin{abstract}
We present a new observable to study halo nuclei. This new observable is a particular ratio of angular distributions for elastic breakup and scattering. For one-neutron halo nuclei, it is shown to be independent of the reaction mechanism and to provide significant information about the structure of the projectile, including binding energy, partial-wave configuration, and radial wave function of the halo. This observable offers new capabilities for the study of nuclear structure far from stability.
\end{abstract}

Keywords: Halo nuclei, angular distribution, elastic scattering, breakup

Nuclear halos are one of the most striking phenomena revealed through the study of extreme states of matter. Their discovery became possible through the development of radioactive beams in the mid 80s [1]. Measured reaction cross sections along an isotopic chain are seen to increase dramatically as the limits of stability are approached as compared with more bound neighboring isotopes. This observation implies that near the end of an isotopic chain (the drip-line), where the neutron number is much larger than the proton number, adding one or two neutrons to a well-bound core may produce a nucleus with a radius much larger than that of its core, suggesting a halo picture for these valence neutrons [2, 3].

The halo phenomenon is believed to arise from the combination of a very low separation energy of the valence particles and the absence of a strong repulsive barrier. For example, this may appear for neutrons loosely bound to a core in an $s$ orbital. The result is a highly delocalized wave function and a considerable probability of finding the valence particles outside the range of their binding interaction to the core, well into the classically forbidden region. It is less likely to find nuclear halos on the proton drip-line or in orbitals involving large angular momentum, as the Coulomb or centrifugal barriers hinder the development

\footnotetext{
*Corresponding author

Email addresses: pierre.capel@centraliens.net (P. Capel), r.johnson@surrey.ac.uk (R. C. Johnson), nunes@nscl.msu.edu (F. M. Nunes)
} 
of the extended wave function. Halo structures have also been observed experimentally in other fields such as atomic and molecular physics [4]. Extreme examples of halo states are Efimov states, an area generating intense activity in molecular physics [5].

These qualitative features of halo states are fairly well established, but ever since the discovery of halo nuclei [1], it has been a challenge to reconcile this picture with the strongly interacting many-fermion structure of real nuclei. On the experimental side much of the difficulty arises because halo nuclei tend to be unstable against the weak interaction and therefore cannot be prepared as a target. Plans are being made to use electron scattering from trapped radioactive ions [6] or from a beam [7] of these exotic species to measure their charge distribution. So far all the available evidence for their structure is obtained indirectly mostly through the measurements of nuclear reactions, such as breakup [8], elastic scattering [9], or knockout [10].

Collisions between a one-neutron halo nucleus and a target can be described as three-body processes in which a projectile $P$, seen as a valence neutron $\mathrm{n}$ loosely bound to a core $c$, impinges on a target $T$. Various theoretical models have been developed to solve the corresponding Schrödinger equation (see Ref. 11] for a review). These models have improved our understanding of the reaction process. They have shown that the mechanisms involved in collisions of loosely-bound nuclei are more complex than initially thought and that extracting information about nuclear structure from reaction measurements is not as straightforward as hoped [12]. Moreover, reaction calculations depend on optical potentials, which describe the interaction between the projectile constituents and the target. These potentials are often unknown. This is especially true for the interaction of the core and the target as there exist little - if any - data to constrain this potential, the core being usually itself radioactive. The uncertainties related to the choice of these potentials hinder the quantitative analysis of experimental data 13]. An observable that is less dependent on the reaction process and that reveals more information about the structure of the projectile is clearly needed. In this Letter, we present such an observable.

In this framework, the structure of the projectile is described by the internal Hamiltonian

$$
H_{0}=\frac{p^{2}}{2 \mu_{c \mathrm{n}}}+V_{c \mathrm{n}}(\boldsymbol{r}),
$$

where $\mu_{c \mathrm{n}}$ is the $c$-n reduced mass, $\boldsymbol{r}$ is the relative $c$-n coordinate, and $\boldsymbol{p}$ the corresponding momentum. The $c$-n potential $V_{c n}$ is adjusted to reproduce properties of the projectile, such as its binding energy and some of its excited levels. In partial wave $l j m$, the eigenstates of $H_{0}$ of energy $E$ are denoted by $\phi_{l j m}(E)$ ( $l$ is the $c$-n orbital angular momentum, $j$ is the total angular momentum resulting from the coupling of $l$ with the neutron spin, and $m$ is the projection of $j$ ). For $E<0$ they are normed to unity and correspond to bound states of the projectile. For $E>0$, they describe the continuum spectrum, i.e. the projectile broken up into $c$ and n. They are normalized as $\left\langle\phi_{l j m}(E) \mid \phi_{l^{\prime} j^{\prime} m^{\prime}}\left(E^{\prime}\right)\right\rangle=\delta_{l l^{\prime}} \delta_{j j^{\prime}} \delta_{m m^{\prime}} \delta\left(E-E^{\prime}\right)$. The interactions of the projec- 
tile fragments $c$ and $n$ with the target are simulated by the optical potentials $V_{c T}$ and $V_{\mathrm{n} T}$, respectively. Within this framework the study of reactions involving one-neutron halo nuclei reduces to solving the three-body Schrödinger equation with Hamiltonian

$$
H=\frac{P^{2}}{2 \mu}+H_{0}+V_{c T}\left(\boldsymbol{R}-\frac{m_{\mathrm{n}}}{m_{P}} \boldsymbol{r}\right)+V_{\mathrm{n} T}\left(\boldsymbol{R}+\frac{m_{c}}{m_{P}} \boldsymbol{r}\right),
$$

where $\mu$ is the $P-T$ reduced mass, $m_{\mathrm{n}}$ is the mass of the valence neutron, and $m_{c}$ that of the core $\left(m_{P}=m_{c}+m_{\mathrm{n}}\right)$. Variable $\boldsymbol{R}$ is the $P-T$ relative coordinate and $\boldsymbol{P}$ the corresponding momentum. The Schrödinger equation corresponding to Hamiltonian (2) must be solved with the condition that the impinging projectile is initially in its ground state $\phi_{0}$.

Recently, within the dynamical eikonal approximation (DEA) [14, 15, 11], angular distributions for the elastic scattering and elastic breakup of one-neutron halo nuclei have been studied [16]. This analysis shows that both processes exhibit very similar features, suggesting that the loosely-bound projectile is scattered similarly whether it remains in its ground state or is broken up. This result can be explained within the Recoil Excitation and Breakup (REB) model [17, 18]. In this model, a simple solution of the three-body Schrödinger equation is obtained by neglecting $V_{\mathrm{n} T}$ and the excitation energy of the projectile (i.e. using the adiabatic - or sudden - approximation). In the REB limit the elasticscattering cross section in direction $\Omega=(\theta, \phi)$ in the $P-T$ center-of-mass rest frame is exactly factorized into the product of an elastic-scattering cross section for a pointlike projectile $(d \sigma / d \Omega)_{\mathrm{pt}}$ and a form factor describing the extension of the halo [17, 18].

The REB model can also describe the angular distributions for excitation of the projectile to any of its states [18, 19]. The corresponding cross sections also factorize into a reaction-dynamics part and a projectile-structure part. In particular, this can be performed for the breakup of the projectile, i.e. its excitation at an energy $E$ in the $c$-n continuum with its center of mass scattered in direction $\Omega$. To the extent that the small difference in magnitude between the outgoing momenta for elastic scattering and breakup can be neglected, the pointlike cross section $(d \sigma / d \Omega)_{\mathrm{pt}}$ is identical in the expression of both processes. This particular feature explains why the angular distributions for elastic scattering and breakup are so similar [16]. It also leads to the main new idea introduced here. It is exactly $(d \sigma / d \Omega)_{\mathrm{pt}}$ that contains the undesired dependence on the $P-T$ relative motion and its sensitivity to $V_{c T}$. Therefore, a ratio of breakup to elastic-scattering angular distributions would naturally remove this dependence and provide information pertaining only to the halo structure. In addition, this observable, being the ratio of two cross sections, would not depend on their absolute normalizations, which is particularly attractive from an experimental point of view.

The same factorization is obtained for the ratio of any linear combination of angular distributions. We have found it optimal to consider the ratio of the angular distribution for elastic breakup at one definite $c$-n relative energy $E$, to the sum of the angular distributions for elastic and inelastic scattering and for 
elastic breakup at all $c$-n energies

$$
\frac{d \sigma_{\mathrm{sum}}}{d \Omega}=\frac{d \sigma_{\mathrm{el}}}{d \Omega}+\frac{d \sigma_{\text {inel }}}{d \Omega}+\int \frac{d^{2} \sigma_{\mathrm{bu}}}{d E d \Omega} d E .
$$

Using the closure relation for the states of the projectile, this ratio is approximated at the REB limit by

$$
\left(\frac{d^{2} \sigma_{\mathrm{bu}} / d E d \Omega}{d \sigma_{\mathrm{sum}} / d \Omega}\right)_{\mathrm{REB}}=\left|F_{E, 0}(\boldsymbol{Q})\right|^{2},
$$

where the form factor reads

$$
\left|F_{E, 0}\right|^{2}=\sum_{l j m}\left|\int \phi_{l j m}(E, \boldsymbol{r}) \phi_{0}(\boldsymbol{r}) e^{i \boldsymbol{Q} \cdot \boldsymbol{r}} d \boldsymbol{r}\right|^{2},
$$

with $\boldsymbol{Q}=\left(m_{\mathrm{n}} / m_{P}\right)\left(\boldsymbol{K}-\boldsymbol{K}^{\prime}\right)$ corresponding to the fraction $m_{\mathrm{n}} / m_{P}$ of the momentum transferred from the incoming $\hbar \boldsymbol{K}$ to the outgoing $\hbar \boldsymbol{K}^{\prime}$ momenta between the center of mass of the $c$-n pair and the target. We show below by comparison with more exact calculations that the REB approximations can be justified in realistic cases. Assuming this temporarily we discuss the structure information that can be extracted from $\left|F_{E, 0}\right|^{2}$.

To illustrate the general properties of $\left|F_{E, 0}\right|^{2}$, we consider ${ }^{11} \mathrm{Be}$ as a projectile. Reactions involving this archetypal one-neutron halo nucleus have been extensively studied both theoretically and experimentally. In particular its angular distributions for breakup on $\mathrm{Pb}$ and $\mathrm{C}$ targets have been precisely measured at about $70 \mathrm{MeV} /$ nucleon [20]. Figs. 1] and 2 depict $\left|F_{E, 0}\right|^{2}$ at $E=0.1 \mathrm{MeV}$ for ${ }^{11}$ Be impinging on lead at $69 \mathrm{MeV} /$ nucleon as a function of the azimuthal angle $\theta$ up to $10^{\circ}$. The form factor (5) is initially computed for the $c$-n potential developed in Ref. [13], in which the $1 / 2^{+}$ground state of ${ }^{11} \mathrm{Be}$ is produced in the $1 s_{1 / 2}$ orbital (solid line in both Figs. 1 1 and 2). The dependence of this form factor on the projectile internal structure is then analyzed. First, in Fig. 1. the sensitivity of $\left|F_{E, 0}\right|^{2}$ to the $c$-n binding energy is investigated by varying the $s$-wave depth of $V_{c n}$ to reproduce the $1 s_{1 / 2}$ ground state at $50 \mathrm{keV}$ (dotted line), the realistic $0.5 \mathrm{MeV}$ (solid line), and $5 \mathrm{MeV}$ (short-dashed line). The dependence on the partial-wave configuration of the ground state is also analyzed in

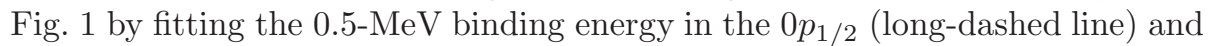
$0 d_{5 / 2}$ (dash-dotted line) orbitals. We observe that both shape and magnitude of the form factor depend strongly on the angular momentum of the valence neutron and its binding energy to the core. This shows that the ratio method could be a very useful tool to measure precisely and simultaneously these two quantities for loosely-bound nuclei, which, such as ${ }^{31} \mathrm{Ne}$ [10], are too short-lived for usual spectroscopic techniques.

Interestingly, the form factor varies also with the shape of the radial part of the ground-state wave function $\phi_{0}$. This point is illustrated in Fig. 2, where the dash-dotted line corresponds to $\left|F_{E, 0}\right|^{2}$ obtained from the $V_{c n}$ of Ref. [13] but with a depth in the $s_{1 / 2}$ partial wave reduced to produce the ground state of 


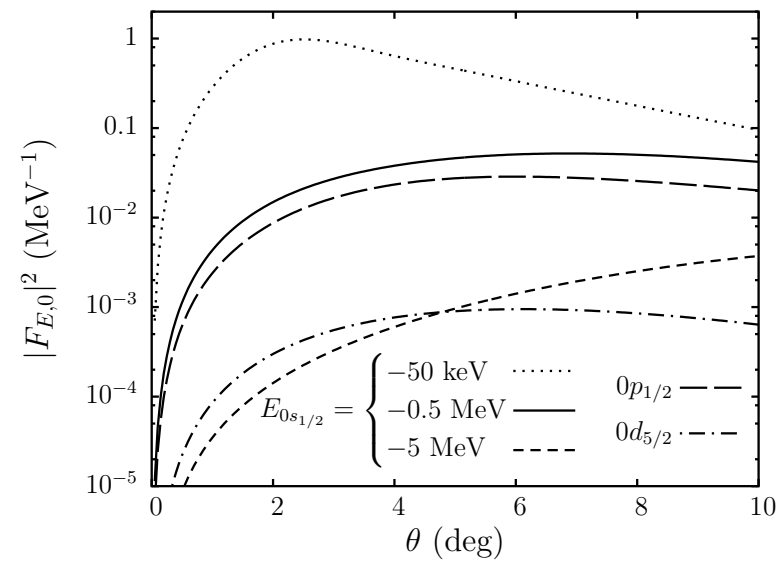

Figure 1: Form factor $\left|F_{E, 0}\right|^{2}$ for ${ }^{11} \mathrm{Be}$ impinging on $\mathrm{Pb}$ at $69 \mathrm{MeV} /$ nucleon. Its sensitivity to the projectile binding energy and partial-wave configuration is illustrated.

${ }^{11} \mathrm{Be}$ in the $0 s_{1 / 2}$ orbital, i.e. without node. We observe interesting changes in the form factor. At forward angles, $\left|F_{E, 0}\right|^{2}$ for the $1 s_{1 / 2}$ bound state is larger than for the $0 s_{1 / 2}$ one. On the contrary, at larger angles, the $0 s_{1 / 2}$ form factor exceeds the $1 s_{1 / 2}$ one. This suggests that depending on the angle considered, $\left|F_{E, 0}\right|^{2}$ probes different parts of the radial wave function. Up to $2^{\circ}$ the form factor scales with the square of the asymptotic normalization coefficient (ANC) that determines the asymptotic exponential form of $\phi_{0}$. The crossing of the form factors shows that larger angles probe the interior of $\phi_{0}$, as in that region the $0 s_{1 / 2}$ wave function, exhibiting no node, is larger than the $1 s_{1 / 2}$ one. Albeit small, the variations observed between both form factors show that the ratio method probes a wider range of the halo wave function than usual breakup observables, which are purely peripheral [21].

The richness of halo-structure information residing in $\left|F_{E, 0}\right|^{2}$ makes the ratio idea particularly attractive. We now address the key question of the accuracy in realistic cases of the REB approximation to the three-body model on which Eq. (4) is based. If the adiabatic treatment of the internal motion of the projectile is lifted and the $\mathrm{n}-T$ interaction is included, does Eq. (4) hold? To answer this question, we make use of the DEA [14, 15] which reproduces closely the data of Ref. 20] and does not make the approximations introduced in the REB model. In this analysis, we use the inputs and numerical conditions detailed in Ref. [15].

In Fig. 3 we present the DEA angular distributions for ${ }^{11} \mathrm{Be}$ impinging on lead at $69 \mathrm{MeV} /$ nucleon. The breakup angular distribution is displayed for a relative $c$-n energy $E=0.1 \mathrm{MeV}$ in $\mathrm{b} / \mathrm{MeV} / \mathrm{sr}$ (dashed line). The summed angular distribution (3) is shown as a ratio to Rutherford (dotted line). As observed in Ref. 16], both exhibit very similar features (Coulomb rainbow, oscillatory patterns,...). In the ratio of these distributions (thin solid line), these features are smoothed out, confirming that most of the dependence on 


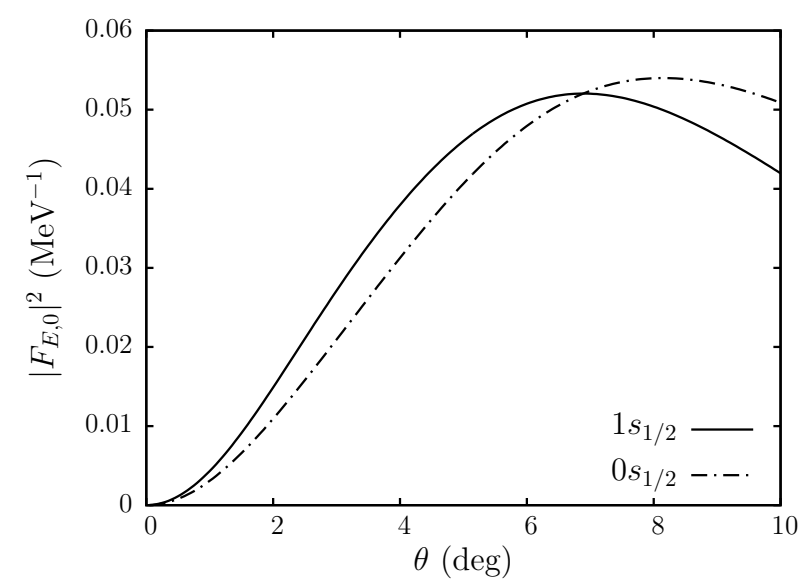

Figure 2: Sensitivity of the form factor $\left|F_{E, 0}\right|^{2}$ to changes in the radial $c$-n wave function. Differences are observed between (realistic) $1 s_{1 / 2}$ and $0 s_{1 / 2}$ ground states.

the reaction dynamics is removed by this technique. Moreover, since the DEA ratio compares very well with its REB prediction $\left|F_{E, 0}\right|^{2}$ (gray thick line), we conclude that the ratio method gives direct access to halo-structure information.

Even though the agreement is generally good, we observe discrepancies between the DEA ratio and $\left|F_{E, 0}\right|^{2}$. In particular, at large angles the former exhibits small oscillations whereas the latter is smooth. To investigate the source of these differences, we repeat the DEA calculation setting $V_{\mathrm{n} T}=0$. We find that the oscillations in the ratio disappear and the agreement with $\left|F_{E, 0}\right|^{2}$ improves. As shown by Johnson et al., $V_{\mathrm{n} T}$ shifts the oscillatory pattern of angular distributions [17]. This shift, depending on the excitation energy of the projectile, brings the breakup and scattering distributions slightly out of phase, causing the oscillations of the ratio in the realistic case. This result indicates that $V_{\mathrm{n} T}$ has non-negligible effects on reaction calculations. However, these effects remain small compared to the dynamical effects removed by the ratio (44).

To evaluate the independence to the $c-T$ interaction gained by the ratio method, we repeat the DEA calculation with different choices of $V_{c T}$. Switching off the nuclear part of that optical potential does not lead to significant changes in the ratio (44), although the characteristics of the angular distributions are significantly altered [16]. The ratio removes the dependence on this interaction, which is a large source of uncertainty in the analysis of experimental data. This result suggests the ratio to be independent of the target, and hence of the reaction mechanism. To confirm this, we compare in Fig. 团 the results on $\mathrm{Pb}$ at $69 \mathrm{MeV} /$ nucleon (thin solid line) with those on $\mathrm{C}$ at $67 \mathrm{MeV} /$ nucleon (dashed line). Now the ratio is plotted as a function of $Q$ to allow the comparison between different targets. Although the reaction mechanism on $\mathrm{C}$ is very different from the Coulomb-dominated collision on $\mathrm{Pb}$, the ratio for ${ }^{11} \mathrm{Be}+\mathrm{C}$ is very close to the ratio for ${ }^{11} \mathrm{Be}+\mathrm{Pb}$. Both are in excellent agreement with $\left|F_{E, 0}\right|^{2}$ (thick 


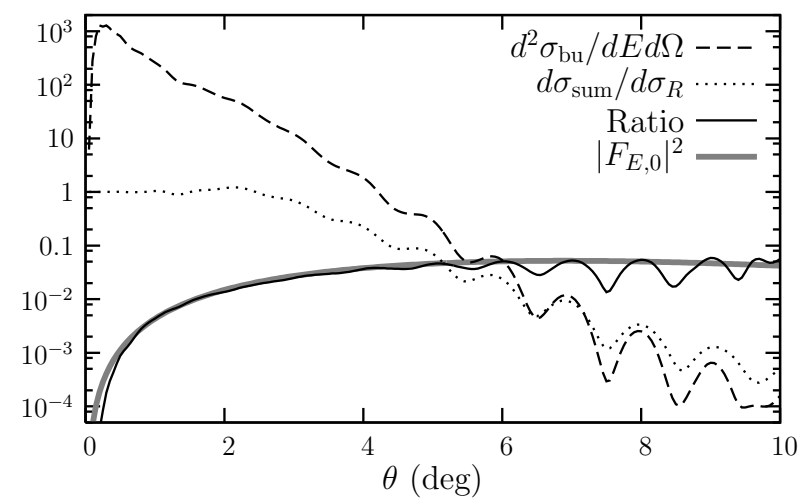

Figure 3: Ratio of the breakup and summed angular distributions for ${ }^{11} \mathrm{Be}$ on $\mathrm{Pb}$ at $69 \mathrm{MeV} /$ nucleon. DEA calculation is compared to its REB estimation $\left|F_{E, 0}\right|^{2}$.

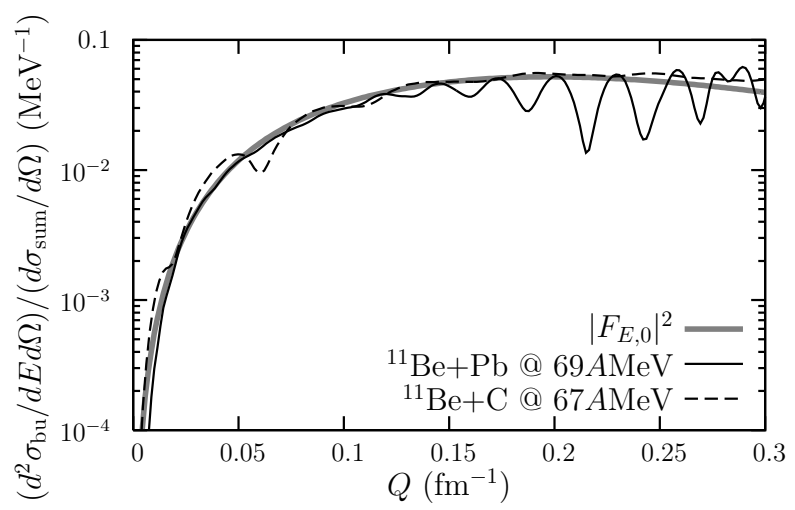

Figure 4: Sensitivity of ratio (4) to the reaction mechanism. DEA calculations on different targets are compared to $\left|F_{E, 0}\right|^{2}$.

gray line). This result shows that besides removing the uncertainty caused by the projectile-target potentials, observable (4) is, for all practical purposes, independent of the reaction mechanism.

In this Letter, we propose the study of the structure of halo nuclei through a new observable consisting in the ratio of two angular distributions: one for the elastic breakup of the projectile at a definite $c$-n energy and the other corresponding to the elastic scattering and elastic breakup of the projectile into any of its states.

We show that this ratio removes the dependence on the reaction mechanism and leads to an observable that can provide detailed information on the halo structure. Besides the binding energy and the angular momentum of the valence neutron, the radial wave function of the halo can be accessed by this ratio. Depending on the angle considered, both asymptotic and internal parts of the wave function can be probed. To assess the influence of a multiconfiguration 
structure of the projectile on this observable, a generalization of the REB model is needed. This will be required to evaluate how spectroscopic factors different from 1 affect the ratio. A discussion on this matter and a detailed study of the sensitivity of the ratio method to the projectile description will be published elsewhere. Derived here for one-neutron halo nuclei, this technique is most likely extendible to multibody systems, such as two-neutron halo nuclei or Efimov states in atomic and molecular physics [4, 5]. An extension to charged valence particles may also be possible [22], which would enable the study of proton halos and halo-like negative ions. This observable looks therefore very promising for the study of loosely-bound quantal structures. At least in the realm of nuclear physics, we believe the ratio method will open a new era in the study of exotic systems.

\section{Acknowledgments}

We thank I. J. Thompson, B. Tsang, N. Timofeyuk, and the MoNA collaboration for interesting discussions on the subject. This work was supported by the National Science Foundation grant PHY-0800026 and the Department of Energy under contract DE-FG52-08NA28552 and de-sc0004087. RCJ is supported by the United Kingdom Science and Technology Facilities Council under Grant No. ST/F012012.

\section{References}

[1] I. Tanihata et al., Phys. Rev. Lett. 55, 2676 (1985).

[2] P. G. Hansen and B. Jonson, Europhys. Lett. 4, 409 (1987).

[3] I. Tanihata, J. Phys. G 22, 157 (1996).

[4] A. S. Jensen, K. Riisager, D. V. Fedorov, and E. Garrido, Rev. Mod. Phys. 76, 215 (2004).

[5] M. Zaccanti et al., Nature Phys. 5, 586 (2009).

[6] T. Suda et al., Phys. Rev. Lett. 102, 102501 (2009).

[7] A. Antonov et al., Nucl. Instrum. Methods Phys. Res. A 637, 60 (2011).

[8] T. Nakamura et al., Phys. Rev. Lett. 96, 252502 (2006).

[9] A. Di Pietro et al., Phys. Rev. Lett. 105, 022701 (2010).

[10] T. Nakamura et al., Phys. Rev. Lett. 103, 262501 (2009).

[11] D. Baye, Eur. Phys. J. Special Topics 156, 93 (2008).

[12] P. Capel and D. Baye, Phys. Rev. C 71, 044609 (2005).

[13] P. Capel, G. Goldstein, and D. Baye, Phys. Rev. C 70, 064605 (2004). 
[14] D. Baye, P. Capel, and G. Goldstein, Phys. Rev. Lett. 95, 082502 (2005).

[15] G. Goldstein, D. Baye, and P. Capel, Phys. Rev. C 73, 024602 (2006).

[16] P. Capel, M. Hussein, and D. Baye, Phys. Lett. B 693, 448 (2010).

[17] R. C. Johnson, J. S. Al-Khalili, and J. A. Tostevin, Phys. Rev. Lett. 79, 2771 (1997).

[18] R. C. Johnson, in Proc. of the Euro. Conf. in Advances in Nucl. Phys. and Related Areas, Thessaloniki, Greece, 8-12 July 1997, edited by D. M. Brink, M. E. Grypeos, and S. E. Massen (Giahoudi-Giapouli Publishing, Thessalonika, 1999), p. 156.

[19] R. C. Johnson, J. Phys. G 24, 1583 (1998).

[20] N. Fukuda et al., Phys. Rev. C 70, 054606 (2004).

[21] P. Capel and F. M. Nunes, Phys. Rev. C 75, 054609 (pages 6) (2007).

[22] K. Horii, M. Takashina, T. Furumoto, Y. Sakuragi, and H. Toki, Phys. Rev. C 81, 061602 (2010). 